\title{
Victimization Experiences and Binge Drinking and Smoking Among Boys and Girls in Grades 7 to 12 in Manitoba, Canada
}

\author{
Shannon Struck ${ }^{1} \cdot$ Janique Fortier $^{1} \cdot$ Samantha Salmon ${ }^{1} \cdot$ Tamara Taillieu $^{1} \cdot$ Tracie O. Afifi $^{2}$ (i)
}

Accepted: 2 November 2020 / Published online: 14 November 2020

(C) The Author(s) 2020

\begin{abstract}
Experiencing victimization, such as cyberbullying, discriminatory harassment, or bullying in adolescence is associated with health risk behaviours. However, inconsistent findings in the literature examining the associations between different types of victimization and binge drinking and smoking exist. This study investigated the association between nine types of victimization experiences and (a) binge drinking and (b) smoking among boys and girls in grades 7 to 12. Data were from the 2012/13 Manitoba Youth Health Survey that included 475 participating schools and 64,174 students. Students in grades 7 to 12 completed the survey at school. Logistic regression models were used to examine the relationships between victimization experiences and binge drinking and smoking. All analyses were stratified by gender and grade groups. All nine types of victimization experiences among boys and girls in grades 7 to 12 were significantly associated with binge drinking and smoking. Overall, a dose-response trend was observed with increasing experiences of victimization related to greater odds of binge drinking and smoking for boys and girls in grades 7 to 9. Findings indicate that specific victimization experiences are associated with increased odds of binge drinking and smoking among adolescents. Prevention efforts to reduce victimization and to help those who have experienced victimization need to be addressed at all grade levels as it may be associated with a reduction in risky behaviours such as binge drinking or smoking among adolescents.
\end{abstract}

Keywords Victimization $\cdot$ Bullying $\cdot$ Adolescents $\cdot$ Binge drinking $\cdot$ Smoking $\cdot$ Discrimination $\cdot$ Cyberbullying

Victimization experiences, including traditional bullying, cyberbullying, and discriminatory harassment, are common among adolescents worldwide (Elgar et al. 2015; Modecki et al. 2014; Salmon et al. 2018) and have been identified as risk factors for future negative physical and mental health outcomes, compromised academic performance, poor interpersonal relationships, and socioeconomic disadvantage (Copeland et al. 2013; Fisher et al. 2016; Grinshteyn and Yang 2017; Herge et al. 2016; Holt et al. 2015; Juvonen et al. 2011; Nakamoto and Schwartz 2010; Sigurdson et al. 2014; Stewart-Tufescu et al. 2019; Takizawa et al. 2014; van Geel et al. 2015; Wolke et al. 2013; Wolke and Lereya 2015).

Tracie O. Afifi

tracie.afifi@umanitoba.ca

1 Department of Community Health Sciences, University of Manitoba, Winnipeg, Canada

2 Departments of Community Health Sciences and Psychiatry, University of Manitoba, S113-750 Bannatyne Avenue, Winnipeg, Manitoba R3E 0W5, Canada
Victimization experienced by adolescents can include traditional bullying, defined as repeat exposure to purposeful, aggressive behaviours by a peer or group of peers with a perceived power imbalance (Modecki et al. 2014; Olweus 2013; Volk et al. 2014). However, adolescents also experience victimization outside of traditionally defined bullying such as single interactions of cyber-victimization and discriminatory harassment (Salmon et al. 2018). Additionally, single exposures to specific victimization experiences have been associated with moderate/languishing mental health functioning and emotional well-being among adolescents (Stewart-Tufescu et al. 2019). Bullying has been associated with risk taking behaviours such as drinking alcohol and smoking (Quinn et al. 2016a, b; Vieno et al. 2011) so it may also be possible that associations between single exposure victimization experiences and substance use such as binge drinking and smoking may also exist. Therefore, more research is warranted to understand the relationship between types of victimization experiences and substance use such as smoking and binge drinking. 
Inconsistent definitions, measurements, and types of victimization have contributed to a range of prevalence estimates. In Canada, a national study found $63 \%$ of youth in grades $6-$ 10 reported experiencing victimization at least once over a 2month period (Freeman et al. 2011). Similarly, a study by Salmon et al. (2018), using the same data used in the current study, indicated that the prevalence of nine specific victimization experiences (e.g., having something bad said about their gender identity, their body shape/appearance, or their race; being physically threatened or injured with a weapon) at least once in the past 12 months ranged from 52.1 to $61.5 \%$ among boys and $57.5-73.3 \%$ among girls in grades 7 to 12 in the Canadian province of Manitoba (Salmon et al. 2018). In contrast, a global study reported that approximately $30 \%$ of youth experienced bullying victimization with an estimated 35-44\% of Canadian 13-year-olds being the target of bullying at least once in the previous 2 months (Elgar et al. 2015). While disparate prevalence estimates may be partially attributed to a lack of established guidelines in measurement, victimization experiences are nonetheless common among youth and continued research in this area is warranted. Research on youth victimization and its associations with health and behavioural outcomes is a growing field of study grappling with establishing common definitions and standard measures that create robust and reproducible findings (Olweus 2013; Volk et al. 2014). Differences in research design and the definition of bullying compared to peer victimization result in conflicting conclusions regarding the prevalence of victimization experiences and their associations with important health risk behaviours such as adolescent binge drinking and smoking (Kert et al. 2010; Napoletano et al. 2015). Inconsistent findings across the literature underscore the importance of continued, and more nuanced, research into this field.

Adolescence is also a time when many youth start experimenting with substance use including binge drinking, typically defined as consuming 4-5 alcoholic drinks in one occasion, and smoking, potentially establishing habits that continue into adulthood with lasting health effects (Freeman et al. 2011; Public Health Agency of Canada 2016). The 2012/ 13 Youth Smoking Survey, a nationally representative study of Canadian adolescents, reported that alcohol remained the most common mind-altering drug used in Canada with $40 \%$ of Canadian youth (grades 6 to 12) reporting using alcohol and 24\% reporting binge drinking in the past 12 months (Freeman et al. 2011; Health Canada 2014). Binge drinking is associated with increased participation in risky sexual behaviours, risky traffic behaviours, and higher consumption of alcohol in adulthood (Freeman et al. 2011). The same report found that the prevalence of having ever tried smoking among adolescents in grades 6 to 12 has been steadily declining from $33 \%$, measured in 2006/2007, to a low of $23 \%$ in 2012/2013 (Freeman et al. 2011; Health Canada 2014). Smoking in adolescence is associated with poor short- and long-term health outcomes such as increased risk of respiratory disease, reduced physical fitness, and an increase risk of lung cancer (World Health Organization 2013). The high prevalence of youth participating in health risk behaviours, such as binge drinking and smoking, is a public health concern. Understanding factors associated with youth binge drinking and smoking is imperative to direct prevention efforts that may, in turn, reduce poor health outcomes later in adulthood in addition to immediate health risks (Health Canada 2014; Public Health Agency of Canada 2016; World Health Organization 2013).

Adolescent victimization has been widely reported as associated with externalizing and negative coping behaviours such as increased substance use including binge drinking and smoking (Fisher et al. 2016; Quinn et al. 2016a, b; Turner et al. 2018; Weiss et al. 2011), but conflicting findings exist (Bouffard and Koeppel 2017; Chan and La Greca 2016), which may lead to inconsistent conclusions. Studies that reported significant associations between various types of victimization experiences (e.g., physical, verbal, relational, homophobic, and cyber) and alcohol used non-specific measures such as ever being drunk or ever using alcohol (Peleg-Oren et al. 2012; Tucker et al. 2016; Vieno et al. 2011). Tucker et al. (2016) investigated the longitudinal association between homophobic name calling and alcohol use and found a positive association between name calling and psychological distress and alcohol use among lesbian, gay, and bisexual students. Other studies examining more specific measures of alcohol use such as binge drinking have reported inconsistent findings (Bouffard and Koeppel 2017; Chan and La Greca 2016). For example, no significant relationship was found between reported peer victimization before age 12 years and binge drinking before the age 18 years for either sex (Bouffard and Koeppel 2017). In contrast, a study among adolescents in grades 9-12 concluded that a significant relationship existed between cyberbullying victimization and binge drinking after controlling for other types of victimization experiences (Chan and La Greca 2016). As such, a more detailed study into the relationship between victimization experiences and binge drinking in adolescents is warranted to clarify if certain specific victimization experiences are associated with increased risk of binge drinking.

Similar inconsistent findings exist in the relationship between victimization experiences and smoking in youth. Significant associations have been established between general and specific victimization experiences (e.g., physical, verbal, relational, and cyber) and current youth smoking with differences noted by age and sex (Azagba 2016; Case et al. 2016; Vieno et al. 2011). Specifically, a dose-response association was noted among Canadian students in grades 9 to 12 between cyber-victimization and increased smoking, meaning that as the frequency of cyberbullying increased, the strength of the relationship to smoking also increased (Chan and La 
Greca 2016). Weiss et al. (2011) investigated the longitudinal effect of bullying on smoking initiation in adolescence and found significant positive associations between bullying victimization and smoking initiation. However, conflicting results were noted in another Canadian study that found no association between peer victimization and smoking, and in an American study that only found an association for girls, but not boys (Bouffard and Koeppel 2017; Morris et al. 2006).

Boys and girls are known to experience victimization differently (Salmon et al. 2018) and experience unique engagement in binge drinking and smoking (Chan and La Greca 2016; Freeman et al. 2011; Sánchez de Ribera et al. 2019). A positive association between interpersonal violence, smoking, and alcohol use was noted among boys but not girls (Sánchez de Ribera et al. 2019); whereas, for girls, an increase in cyberbullying was related to increased drinking but the same relationship was not found for boys (Chan and $\mathrm{La}$ Greca 2016). Furthermore, there is evidence that sex plays a role in the association between racial discrimination and smoking; boys who experienced racial discrimination had higher odds of smoking, whereas as their female peers that experienced racial discrimination did not (Agunwamba et al. 2017; Assari et al. 2018). Similarly, experiences of victimization and as well as the associated health risk behaviours such as substance use, smoking, and binge drinking vary depending on an adolescents age and grade (Azagba 2016; Boyce 2004; Salmon et al. 2018; Turner et al. 2018). In particular, younger middle school teens were more susceptible to start smoking when compared to older teens (Azagba 2016) and grade differences were noted with the associations between peer victimization and substance use (Turner et al. 2018). The above findings reinforce the need to study victimization by type and exposure stratified by sex and age or grade group to understand the nuanced experiences of victimization and substance use among youth.

The current literature on the associations between victimization experiences and binge drinking and smoking among youth are limited by the following: nonrepresentative samples with narrow age ranges (Case et al. 2016; Quinn et al. 2016a, b; Vieno et al. 2011), measuring only one type of victimization (Fisher et al. 2016; Kowalski et al. 2014; Rosenthal et al. 2015), not stratifying by sex (Chan and La Greca 2016; Peleg-Oren et al. 2012; van Geel et al. 2015), and non-specific bullying or peer victimization questions (Azagba 2016; Bouffard and Koeppel 2017). The current study addresses the above limitations with the use of a large school-based sample to measure nine specific victimization experiences, stratified by sex and grade. The main objectives of the current study were to determine if nine different experiences of victimization in youth were associated with an increased likelihood of (1) binge drinking and (2) smoking among boys and girls in grade 7 to 12 in Manitoba, Canada, and (3) to observe if dose-response trends were noted with increasing exposure to victimization associated with increasing odds of both binge drinking and smoking.

\section{Data and Sample}

Data were collected using a self-administered paper survey as part of the second cycle of the Manitoba Youth Health Survey during the 2012/2013 academic year. All school divisions across the province were invited to administer the survey to students in grade 7 to 12 . Participation was voluntary and parental consent was obtained according to local school division policies. A total of 64,174 grade 7 to 12 students from 475 schools, including public, independent, Francophone, Colony, and First Nations schools, completed the survey (population response rate $=67 \%$ of students and $73 \%$ of schools) (Manitoba Youth Health Survey Report//20122013 2014). Partners in Planning for Healthy Living, a network of government, non-government, and Regional Health Authorities in Manitoba, supported the Manitoba Youth Health Survey. Ethical approval for the current study of secondary data from the Manitoba Youth Health Survey was obtained from the Health Research Ethics Board at the University of Manitoba. Details on survey implementation and the socio-demographics of student participants have been published elsewhere (Manitoba Youth Health Survey Report//2012-2013 2014; Salmon et al. 2018; Turner et al. 2018).

\section{Measures}

\section{Victimization Experiences}

To assess victimization, respondents were asked to report how many times in the past year (12 months) they had experienced any of the following: (1) were physically threatened or injured; (2) were threatened or injured with a weapon such as a gun, knife, or club; (3) were bullied, taunted, or ridiculed; (4) someone said something bad about their race or culture; (5) someone said something bad about their sexual orientation or gender identity; (6) someone said something bad about their body shape, size, or appearance; (7) someone asked for personal information over the Internet (e.g., address, phone number, or last name); (8) someone made them feel unsafe when in contact over the internet; and (9) were bullied or picked on through the internet (e.g., posted something on Facebook or by email). Response categories included (1) never, (2) 1 to 5 times, (3) 6 or more times, and (4) every day. The internal consistency of the victimization questions was acceptable $(\alpha=0.77)$. 


\section{Binge Drinking}

Binge drinking was assessed with the following question: "During the past month (30 days), on how many days did you have five or more drinks of alcohol within a couple of hours?". Response categories included (1) I have never drank alcohol; (2) I have drank alcohol, but not in the past month; (3) 1 or 2 days, (4) 3 to 5 days; (5) 6 to 9 days; and (6) 10 or more days. These categories were further dichotomized in yes or no categories where a response of one or more days was coded as yes.

\section{Smoking}

Smoking was assessed with the following question: "During the past month (30 days), did you smoke a cigarette, even just a few puffs?". Response categories included (1) yes, every day; (2) yes, almost every day; (3) yes, some days; and (4) no. These categories were further dichotomized in yes or no categories.

\section{Statistical Analyses}

The sample was stratified by sex (boys and girls) and by grade groups (grades 7 to 9 and grades 10 to 12) creating four subgroups of students: boys (grades 7-9), boys (grades 10-12), girls (grades 7-9), and girls (grades 10-12). These grade grouping were chosen to represent middle school (grades 7 9) and senior school (grades 10-12) and to allow for similar sized sub-samples that remained large enough to provide adequate power to stratify by sex. The prevalence of binge drinking and smoking was computed for each of the four subgroups. Logistic regression analyses were used to examine the relationships between the frequency of specific victimization experiences (never, 1-5 times, $6+$ times, and every day) and (a) binge drinking and (b) smoking, separately for each of the four sub-groups listed above.

\section{Results}

The prevalence of past-month binge drinking and smoking among boys and girls in grades 7 to 9 and in grades 10 to 12 are presented in Table 1 . The prevalence of the specific victimization experiences have been previously published (Salmon et al. 2018).Table 2 presents the results of logistic regression analyses examining the relationship between each type of victimization and binge drinking among the four sample sub-groups. All victimization experiences were associated with increased odds of past-month binge drinking among boys and girls in grades 7 to 9 and grades 10 to 12, with few exceptions. The experience of being bullied, taunted, or ridiculed was not significantly associated to binge drinking until the highest frequency of everyday exposure for boys in grades 10 to 12 . Similarly, having had something bad said about their race/culture was significantly associated with binge drinking only at a larger frequency level for both boys and girls in the older grade groups. As the frequency of experiencing victimization increased, the odds of binge drinking increased for boys and girls in all grade groups. Table 3 presents the associations between each type of victimization and past-month smoking among boys and girls in grades 7 to 9 and grades 10 to 12 . All victimization experiences were associated with smoking, apart from the experience of being bullied, taunted, or ridiculed 1 to 5 times in the past 12 months among boys across grades 7 to 12 . As the frequency of experiencing victimization increased, the odds of smoking increased for each of the relationships studied.

\section{Discussion}

The present study advances knowledge on adolescent victimization experiences with the following novel findings. First, all types of victimization were associated with increased odds of binge drinking and smoking among boys and girls in grades 7 to 9 and grades 10 to 12 . Second, this study found that boys in grades 7 to 9 who experienced victimization daily had the highest increased odds of both binge drinking and smoking suggesting a potential priority area for prevention efforts. Third, there were increasing odds of binge drinking and smoking as the frequency of most types of victimization increased resembling dose-response type trends. For both boys and girls in grades 7 to 9 and 10 to 12 , however, more robust trends were found for those in grades 7 to 9 .

This study supports prior research establishing an association between victimization experiences and binge drinking and smoking in adolescence (Fisher et al. 2016; Quinn et al. 2016a, b; Weiss et al. 2011) and extends the current knowledge base by investigating nine specific victimization experiences stratified by grade group and sex to provide for more detailed information about the relationships. Some studies in the literature did not define the type of victimization (Azagba 2016) or only focused on limited age groups (Case et al. 2016; Quinn et al. 2016a, b; Vieno et al. 2011) when investigating the relationship between adolescent victimization and binge drinking or smoking. Assumptions cannot be made that these experiences and related health risk behaviours will be the same across sex and grade. By asking about specific victimization experiences, this study provides reproducible results. Furthermore, understanding these relationships with greater specificity may allow for more targeted actions for prevention of victimization which, although causal relationships cannot be inferred, may in turn result in a reduction of risky health behaviours such as binge drinking and smoking. 
Table 1 Prevalence of binge drinking and smoking among boys and girls in grades 7 to 9 and 10 to 12 in Manitoba

\begin{tabular}{|c|c|c|c|c|}
\hline \multirow{2}{*}{$\begin{array}{l}\text { Binge drinking and } \\
\text { smoking }\end{array}$} & \multicolumn{2}{|l|}{ Boys } & \multicolumn{2}{|l|}{ Girls } \\
\hline & $\begin{array}{l}\text { Grades } 7-9 \\
(n=17,872) \\
\%(n)\end{array}$ & $\begin{array}{l}\text { Grades } 10-12 \\
(n=15,040) \\
\%(n)\end{array}$ & $\begin{array}{l}\text { Grades } 7-9 \\
(n=16,768) \\
\%(n)\end{array}$ & $\begin{array}{l}\text { Grades } 10-12 \\
(n=14,494) \\
\%(n)\end{array}$ \\
\hline \multicolumn{5}{|l|}{ Binge drinking } \\
\hline No & $94.4(15,693)$ & $68.5(9691)$ & $94.0(14,958)$ & 69.3 (9574) \\
\hline Yes & $5.7(940)$ & $31.5(4459)$ & $6.0(959)$ & 30.7 (4249) \\
\hline \multicolumn{5}{|l|}{ Smoker } \\
\hline No & $94.6(16,898)$ & $81.1(12,195)$ & $94.0(15,757)$ & $83.4(12,081)$ \\
\hline Yes & $5.5(974)$ & $18.9(2845)$ & $6.0(1011)$ & 16.7 (2413) \\
\hline
\end{tabular}

While the data in this study did not allow for determining the underlying mechanism for the associations, binge drinking and smoking may be maladaptive strategies for coping with the negative emotions associated with experiencing victimization. Weiss et al. (2011) determined that hostility and depressive symptoms combined with peer victimization increased the odds of initiating smoking and, therefore, programs that teach students coping strategies to manage emotions could possibly reduce the prevalence of smoking initiation (Weiss et al. 2011). It is possible that the same logic could be applied to experiencing victimization and binge drinking behaviour. Further research in the area is required.

The findings of this study should be considered with several limitations in mind. First, as with all secondary data analyses, our study was constrained by the available data and, thus, we were unable to access other important covariates that might influence the relationship between victimization and adolescent substance use. As well, the perceived power imbalance between the victim and perpetrator, or the type of the perpetrator (e.g., peer, parent, sibling)—both key components in any victimization research (Olweus 2013) — were not measured in the Manitoba Youth Health Survey. Therefore, this study investigated specific adolescent victimization experiences and not bullying as it is traditionally defined. The available data also did not allow for the examination of other roles such as perpetrator, bystander, or defender, which have been found to mediate the relationship between peer victimization and substance use (Quinn et al. 2016a, b). However, the detailed investigation of specific victimization experiences provides novel findings and future studies may build upon these findings to include specific experiences of perpetrators, bystanders, and defenders allowing for a greater understanding of youth victimization. As well, the data used in this study were from the most recent provincial-level study to investigate victimization experiences and substance use among adolescents in Manitoba. However, as the data were collected several years ago, it may not reflect the current experiences of youth in Manitoba Despite the age of the data, the findings still represent novel insight into the associations between unique victimization experiences and binge drinking and smoking among adolescents in Manitoba. Additionally, low prevalence of "every day" frequencies contributed to wide confidence intervals and should be interpreted with caution. It should also be noted that the frequency options available for reporting victimization overlapped. It is possible that some students chose "6+ times" to represent "daily" and therefore, there may be some misclassification and findings should be interpreted with caution. The Manitoba Youth Health Survey was developed in consultation with experts; however, as no formal validation studies of the survey have been done, the results must be interpreted with this in mind. Finally, given the cross-sectional nature of the data, causal associations cannot be inferred, and we cannot eliminate the possibility of an inverse relationship.

This study provides evidence that supports the association between victimization experiences and binge drinking and smoking among boys and girls in grades 7 to 12 . The positive dose-response trends suggest that efforts to prevent or reduce adolescent victimization may be associated with reductions in binge drinking and smoking among adolescents. The large effect sizes for younger students suggest that formal prevention efforts aimed at reducing and preventing victimization and substance use should begin before grade 7 . Further research and efforts to reduce bullying and other detrimental victimization experiences, which may also decrease the likelihood of substance use among adolescents, are warranted. Educators and public health officials working on such programs need to be aware of increased likelihood of substance use among adolescents who experience victimization. Traditional bullying prevention programs need to consider the mounting evidence that victimization experiences that fall just beyond the traditional definition of bullying are also associated with poor outcomes (Stewart-Tufescu et al. 2019; Turner et al. 2018). This study supports other calls for crosscurricular programs addressing both substance use and bullying/victimization to better support the healthy 
Table 2 The relationship between each bullying victimization experience and binge drinking among boys and girls in grades 7 to 12 in Manitoba

\begin{tabular}{lll}
\hline Type of bullying & Boys \\
\cline { 2 - 3 } & Grades 7-9 & Grades 10-12 \\
OR (95\% CI) & OR (95\% CI)
\end{tabular}

Girls

Grades 7-9

OR $(95 \% \mathrm{CI})$

Grades $10-12$

OR $(95 \% \mathrm{CI})$

Physically threatened or injured you

$\begin{array}{lllll}\text { Never } & 1.00 & 1.00 & 1.00 & 1.00 \\ 1-5 \text { times } & 2.04(1.75-2.37) & 1.72(1.59-1.87) & 4.00(3.47-4.62) & 2.17(1.99-2.37) \\ 6+\text { times } & 3.12(2.42-4.02) & 1.85(1.55-2.20) & 5.28(4.06-6.88) & 2.09(1.70-2.58) \\ \text { Everyday } & 9.78(6.89-13.88) & 3.85(2.99-4.96) & 8.75(5.22-14.69) & 2.39(1.44-3.97)\end{array}$

Threatened or injured you with a weapon such as a gun, knife, or club

$\begin{array}{lllll}\text { Never } & 1.00 & 1.00 & 1.00 & 1.00 \\ 1-5 \text { times } & 5.11(4.23-6.18) & 2.70(2.38-3.06) & 8.09(6.60-9.93) & 2.63(2.18-3.17) \\ \text { 6+ times } & 8.15(5.32-12.50) & 3.58(2.59-4.93) & 8.68(5.20-14.48) & 2.71(1.59-4.64) \\ \text { Everyday } & 31.45(19.65-50.35) & 6.88(4.85-9.78) & 5.06(1.41-18.17) & 3.12(1.31-7.40)\end{array}$

Bullied, taunted, or ridiculed you

$\begin{array}{lllll}\text { Never } & 1.00 & 1.00 & 1.00 & 1.00 \\ 1-5 \text { times } & 1.20(1.02-1.41) & 1.06(0.97-1.15) & 1.99(1.71-2.32) & 1.38(1.27-1.50) \\ 6+\text { times } & 1.36(1.07-1.73) & 1.02(0.89-1.17) & 2.81(2.32-3.41) & 1.63(1.44-1.84) \\ \text { Everyday } & 3.92(2.95-5.22) & 1.90(1.56-2.33) & 3.77(2.87-4.95) & 1.53(1.21-1.94)\end{array}$

Said something bad about your race or culture

$\begin{array}{lllll}\text { Never } & 1.00 & 1.00 & 1.00 & 1.00 \\ 1-5 \text { times } & 1.36(1.12-1.64) & 1.09(0.99-1.21) & 1.66(1.40-1.97) & 1.01(0.91-1.11) \\ \text { 6+ times } & 2.31(1.76-3.03) & 1.10(0.93-1.30) & 3.42(2.70-4.34) & 1.22(1.02-1.45) \\ \text { Everyday } & 8.11(6.08-10.82) & 2.17(1.78-2.64) & 4.08(2.72-6.10) & 1.96(1.43-2.68)\end{array}$

Said something bad about your sexual orientation or gender identity

$\begin{array}{lllll}\text { Never } & 1.00 & 1.00 & 1.00 & 1.00 \\ 1-5 \text { times } & 2.02(1.61-2.53) & 1.33(1.16-1.52) & 2.58(2.14-3.13) & 1.53(1.35-1.74) \\ 6+\text { times } & 2.62(1.84-3.75) & 1.26(1.00-1.60) & 3.95(2.95-5.28) & 2.13(1.72-2.63) \\ \text { Everyday } & 11.72(8.36-16.41) & 2.95(2.30-3.78) & 4.70(2.98-7.40) & 2.59(1.75-3.85)\end{array}$

Said something bad about your body shape, size, or appearance

$\begin{array}{lllll}\text { Never } & 1.00 & 1.00 & 1.00 & 1.00 \\ 1-5 \text { times } & 1.41(1.18-1.67) & 1.36(1.24-1.48) & 2.31(1.97-2.71) & 1.70(1.57-1.85) \\ \text { 6+ times } & 1.82(1.41-2.34) & 1.31(1.13-1.52) & 3.30(2.72-4.00) & 2.04(1.82-2.29) \\ \text { Everyday } & 5.87(4.51-7.63) & 2.47(2.03-3.02) & 5.12(4.03-6.51) & 1.82(1.50-2.21)\end{array}$

Asked for personal information over the Internet (e.g., address, phone \#, or last name)

$\begin{array}{lllll}\text { Never } & 1.00 & 1.00 & 1.00 & 1.00 \\ 1-5 \text { times } & 2.41(2.00-2.89) & 1.37(1.23-1.52) & 3.17(2.71-3.69) & 1.60(1.46-1.74) \\ \text { 6+ times } & 4.49(3.35-6.01) & 1.86(1.54-2.24) & 6.98(5.63-8.66) & 2.38(2.05-2.75) \\ \text { Everyday } & 22.50(15.16-33.38) & 4.62(3.48-6.14) & 11.17(7.56-16.49) & 3.28(2.26-4.76)\end{array}$

Made you feel unsafe when you were in contact with them over the Internet

$\begin{array}{lllll}\text { Never } & 1.00 & 1.00 & 1.00 & 1.00 \\ 1-5 \text { times } & 2.35(1.83-3.01) & 1.40(1.18-1.67) & 2.73(2.30-3.24) & 1.37(1.22-1.53) \\ 6+\text { times } & 4.32(2.61-7.15) & 1.80(1.21-2.66) & 5.38(4.09-7.08) & 1.82(1.45-2.30) \\ \text { Everyday } & 30.58(19.14-48.86) & 6.31(4.39-9.07) & 5.69(3.47-9.30) & 2.04(1.27-3.29)\end{array}$

Bullied or picked on you through the Internet (e.g., posted something on Facebook or emailed you)

\begin{tabular}{lllll} 
Never & 1.00 & 1.00 & 1.00 & 1.00 \\
$1-5$ times & $2.31(1.86-2.86)$ & $1.40(1.22-1.61)$ & $2.77(2.36-3.25)$ & $1.80(1.63-1.99)$ \\
6+ times & $3.85(2.69-5.52)$ & $1.51(1.14-2.00)$ & $4.17(3.36-5.18)$ & $2.07(1.75-2.46)$ \\
Everyday & $17.56(11.90-25.91)$ & $4.93(3.59-6.77)$ & $6.48(4.72-8.91)$ & $1.65(1.17-2.34)$ \\
\hline
\end{tabular}

OR, odds ratio; $C I$, confidence interval 
Table 3 The relationship between each bullying victimization experiences and smoking among boys and girls in grades 7 to 12 in Manitoba

\begin{tabular}{|c|c|c|c|c|}
\hline \multirow[t]{2}{*}{ Type of Bullying } & \multicolumn{2}{|l|}{ Boys } & \multicolumn{2}{|l|}{ Girls } \\
\hline & $\begin{array}{l}\text { Grades } 7-9 \\
\text { OR }(95 \% \text { CI) }\end{array}$ & $\begin{array}{l}\text { Grades } 10-12 \\
\text { OR }(95 \% \mathrm{CI})\end{array}$ & $\begin{array}{l}\text { Grades } 7-9 \\
\text { OR }(95 \% \mathrm{CI})\end{array}$ & $\begin{array}{l}\text { Grades } 10-12 \\
\text { OR }(95 \% \mathrm{CI})\end{array}$ \\
\hline \multicolumn{5}{|c|}{ Physically threatened or injured you } \\
\hline Never & 1.00 & 1.00 & 1.00 & 1.00 \\
\hline $1-5$ times & $2.30(1.97-2.67)$ & $1.93(1.76-2.12)$ & $4.70(4.08-5.40)$ & $3.33(3.02-3.68)$ \\
\hline $6+$ times & $3.55(2.77-4.55)$ & $2.56(2.21-3.08)$ & $5.20(3.98-6.79)$ & $3.98(3.22-4.94)$ \\
\hline Everyday & $12.47(8.93-17.42)$ & $6.03(4.72-7.71)$ & $6.62(3.80-11.55)$ & $7.49(4.56-12.32)$ \\
\hline \multicolumn{5}{|c|}{ Threatened or injured you with a weapon such as a gun, knife, or club } \\
\hline Never & 1.00 & 1.00 & 1.00 & 1.00 \\
\hline $1-5$ times & $6.48(5.40-7.77)$ & $3.94(3.47-4.48)$ & $8.50(6.96-10.39)$ & $5.42(4.51-6.53)$ \\
\hline $6+$ times & $10.45(6.97-15.68)$ & $5.16(3.78-7.06)$ & $11.12(6.81-18.16)$ & $7.47(4.45-12.55)$ \\
\hline Everyday & $43.52(27.26-69.50)$ & $9.06(6.64-12.37)$ & $10.45(3.49-31.27)$ & $9.61(4.03-22.94)$ \\
\hline \multicolumn{5}{|c|}{ Bullied, taunted, or ridiculed you } \\
\hline Never & 1.00 & 1.00 & 1.00 & 1.00 \\
\hline $1-5$ times & $1.12(0.94-1.32)$ & $1.02(0.92-1.3)$ & $1.78(1.53-2.08)$ & $1.42(1.29-1.58)$ \\
\hline $6+$ times & $1.67(1.34-2.09)$ & $1.20(1.02-1.40)$ & $2.67(2.21-3.23)$ & $1.96(1.71-2.25)$ \\
\hline Everyday & $4.59(3.49-6.03)$ & $3.40(2.78-4.15)$ & $3.93(3.03-5.11)$ & $3.29(2.59-4.18)$ \\
\hline \multicolumn{5}{|c|}{ Said something bad about your race or culture } \\
\hline Never & 1.00 & 1.00 & 1.00 & 1.00 \\
\hline $1-5$ times & $1.98(1.66-2.37)$ & $1.30(1.16-1.46)$ & $2.79(2.39-3.26)$ & $1.65(1.47-1.84)$ \\
\hline $6+$ times & $2.88(2.21-3.74)$ & $1.56(1.31-1.87)$ & $4.18(3.31-5.28)$ & $2.12(1.76-2.57)$ \\
\hline Everyday & $10.26(7.76-13.55)$ & $3.64(2.99-4.43)$ & $7.09(4.98-10.11)$ & $4.18(3.04-5.73)$ \\
\hline
\end{tabular}

Said something bad about your sexual orientation or gender identity

$\begin{array}{lllll}\text { Never } & 1.00 & 1.00 & 1.00 & 1.00 \\ 1-5 \text { times } & 2.23(1.79-2.78) & 1.38(1.19-1.61) & 3.59(3.01-4.28) & 2.22(1.94-2.54) \\ \text { 6+ times } & 3.71(2.69-5.11) & 2.24(1.77-2.85) & 6.01(4.62-7.81) & 3.06(2.46-3.81) \\ \text { Everyday } & 13.48(9.71-18.71) & 4.74(3.72-6.05) & 9.76(6.62-14.41) & 6.09(4.12-8.98) \\ \text { Said something bad about your body shape, size, or appearance } & & 1.00 \\ \text { Never } & 1.00 & 1.00 & 1.00 & 1.77(1.60-1.96) \\ 1-5 \text { times } & 1.40(1.18-1.67) & 1.34(1.21-1.48) & 2.19(1.87-2.56) & 2.63(2.30-3.00) \\ \text { 6+ times } & 1.91(1.49-2.45) & 1.37(1.16-1.62) & 3.01(2.48-3.66) & 3.36(2.73-4.14) \\ \text { Everyday } & 5.91(4.55-7.67) & 3.84(3.14-4.69) & 6.06(4.84-7.59) & \end{array}$

Asked for personal information over the Internet (e.g., address, phone \#, or last name)

$\begin{array}{lllll}\text { Never } & 1.00 & 1.00 & 1.00 & 1.00 \\ 1-5 \text { times } & 2.63(2.20-3.16) & 1.46(1.30-1.65) & 2.61(2.24-3.05) & 1.60(1.44-1.78) \\ 6+\text { times } & 4.47(3.33-6.00) & 2.23(1.83-2.72) & 5.33(4.28-6.64) & 2.71(2.31-3.18) \\ \text { Everyday } & 23.81(16.15-35.09) & 6.43(4.92-8.40) & 10.02(6.80-14.76) & 5.60(3.88-8.08)\end{array}$

Made you feel unsafe when you were in contact with them over the Internet

$\begin{array}{lllll}\text { Never } & 1.00 & 1.00 & 1.00 & 1.00 \\ 1-5 \text { times } & 3.32(2.65-4.17) & 1.86(1.54-2.23) & 2.73(2.30-3.23) & 1.76(1.55-2.00) \\ \text { 6+ times } & 8.02(5.19-12.4) & 2.90(1.95-4.32) & 5.84(4.47-7.61) & 3.16(2.50-4.01) \\ \text { Everyday } & 33.47(21.25-52.72) & 11.48(8.10-16.26) & 5.38(3.26-8.87) & 6.09(3.81-9.72)\end{array}$

Bullied or picked on you through the internet (e.g., posted something on Facebook or emailed you)

\begin{tabular}{lllll} 
Never & 1.00 & 1.00 & 1.00 & 1.00 \\
$1-5$ times & $3.52(2.90-4.27)$ & $1.77(1.53-2.06)$ & $3.20(2.73-3.75)$ & $2.21(1.98-2.48)$ \\
$6+$ times & $4.53(3.18-6.44)$ & $2.20(1.65-2.95)$ & $5.74(4.69-7.02)$ & $3.58(3.00-4.27)$ \\
Everyday & $24.98(17.23-36.22)$ & $9.04(6.66-12.27)$ & $9.67(7.20-12.98)$ & $4.29(3.05-6.04)$ \\
\hline
\end{tabular}

$O R$, odds ratio; $C I$, confidence interval 
development of adolescents (Hirschtritt 2020). Such programs may consider including references to examples of specific victimization experiences, such as those included in this study. The findings from this study highlight the importance of preventing victimization which in turn, may potentially reduce substance use and at-risk health behaviours among youth.

Acknowledgements The authors would like to acknowledge Lin Xue and Elizabeth Harland of CancerCare Manitoba for their assistance with statistical analyses. The authors would like to also acknowledge Sarah Turner for her efforts in the early planning stage of this project.

Funding Preparation of this article was supported by a Canadian Institutes of Health Research (CIHR) New Investigator Award (Afifi), a CIHR Foundation Grant (Afifi), and a Research Manitoba (Formerly Manitoba Health Research Council) Establishment Award (Afifi).

\section{Compliance with Ethical Standards}

Conflict of Interest The authors declare that they have no conflict of interest.

Open Access This article is licensed under a Creative Commons Attribution 4.0 International License, which permits use, sharing, adaptation, distribution and reproduction in any medium or format, as long as you give appropriate credit to the original author(s) and the source, provide a link to the Creative Commons licence, and indicate if changes were made. The images or other third party material in this article are included in the article's Creative Commons licence, unless indicated otherwise in a credit line to the material. If material is not included in the article's Creative Commons licence and your intended use is not permitted by statutory regulation or exceeds the permitted use, you will need to obtain permission directly from the copyright holder. To view a copy of this licence, visit http://creativecommons.org/licenses/by/4.0/.

\section{References}

Agunwamba, A. A., Kawachi, I., Williams, D. R., Finney Rutten, L. J., Wilson, P. M., \& Viswanath, K. (2017). Mental health, racial discrimination, and tobacco use differences across rural-urban California. The Journal of Rural Health, 33(2), 180-189. https:// doi.org/10.1111/jrh.12182.

Assari, S., Mistry, R., \& Caldwell, C. H. (2018). Perceived discrimination and substance use among caribbean black youth; gender differences. Brain Sciences, 8(7), 1-17. https://doi.org/10.3390/ brainsci8070131.

Azagba, S. (2016). School bullying and susceptibility to smoking among never-tried cigarette smoking students. Preventive Medicine, 85, 69-73. https://doi.org/10.1016/j.ypmed.2016.01.006.

Bouffard, L. A., \& Koeppel, M. D. H. (2017). Sex differences in the health risk behavior outcomes of childhood bullying victimization. Victims \& Offenders, 12(4), 549-565. https://doi.org/10.1080/ 15564886.2015.1118420.

Boyce, W. F. (2004). Young people in Canada : their health and wellbeing 2001-2002 survey. Ottawa.

Case, K. R., Cooper, M., Creamer, M. L., Mantey, D., \& Kelder, S. (2016). Victims of bullying and tobacco use behaviors in adolescents: differences between bullied at school, electronically, or both. Journal of School Health, 86(11), 832-840. https://doi.org/10.1111/ josh.12437.
Chan, S. F., \& La Greca, A. M. (2016). Cyber victimization and aggression: are they linked with adolescent smoking and drinking? Child \& Youth Care Forum, 45(1), 47-63. https://doi.org/10.1007/ s10566-015-9318-x.

Copeland, W. E., Wolke, D., Angold, A., \& Costello, E. J. (2013). Adult psychiatric outcomes of bullying and being bullied by peers in childhood and adolescence. JAMA Psychiatry, 70(4), 419-426. https:// doi.org/10.1001/jamapsychiatry.2013.504.

Elgar, F. J., McKinnon, B., Walsh, S. D., Freeman, J., Donnelly, P. D., De Matos, M. G., et al. (2015). Structural determinants of youth bullying and fighting in 79 countries. Journal of Adolescent Health, 57(6), 643-650. https://doi.org/10.1016/j.jadohealth.2015.08.007.

Fisher, B. W., Gardella, J. H., \& Teurbe-Tolon, A. R. (2016). Peer cybervictimization among adolescents and the associated internalizing and externalizing problems: a meta-analysis. Journal of Youth and Adolescence, 45(9), 1727-1743. https://doi.org/10.1007/ s10964-016-0541-z.

Freeman, J. G., King, M., Pickett, W., Craig, W., Elgar, F., Janssen, I., \& Klinger, D. (2011). The health of Canada's young people: a mental health focus. Ottawa, ON. Retrieved from http://educ.queensu.ca. uml.idm.oclc.org/sites/webpublish.queensu.ca.educwww/files/files/ Research/SPEG/SPEG The Health of Canada's Young People A Mental Health Focus.pdf. Accessed 2 April 2018.

Grinshteyn, E., \& Yang, T. (2017). The association between electronic bullying and school absenteeism among high school students in the United States. Journal of School Health, 87(2), 142-149. https://doi. org/10.1111/josh.12503.

Health Canada. (2014). Summary of results of the youth smoking survey 2012-2013 - Canada.ca. Retrieved May 2, 2018, from https://www. canada.ca/en/health-canada/services/publications/healthy-living/ summary-results-youth-smoking-survey-2012-2013.html. Accessed 22 March 2018.

Herge, W. M., La Greca, A. M., \& Chan, S. F. (2016). Adolescent peer victimization and physical health problems. Journal of Pediatric Psychology, 41(1), 15-27. https://doi.org/10.1093/jpepsy/jsv050.

Hirschtritt, M. E. (2020). Are substance use and bullying perpetration two sides of the same coin? Journal of Adolescent Health, 67, 311-312. https://doi.org/10.1016/j.jadohealth.2020.05.054.

Holt, M. K., Vivolo-Kantor, A. M., Polanin, J. R., Holland, K. M., DeGue, S., Matjasko, J. L., Wolfe, M., \& Reid, G. (2015). Bullying and suicidal ideation and behaviors: a meta-analysis. Pediatrics, 135(2), e496-e509. https://doi.org/10.1542/peds.20141864.

Juvonen, J., Wang, Y., \& Espinoza, G. (2011). Bullying experiences and compromised academic performance across middle school grades. Journal of Early Adolescence, 31(1), 152-173. https://doi.org/10. 1177/0272431610379415.

Kert, A. S., Codding, R. S., Tryon, G. S., \& Shiyko, M. (2010). Impact of the word "bully" on the reported rate of bullying behavior. Psychology in the Schools, 47(2), 193-204. https://doi.org/10. 1002/pits.20464.

Kowalski, R. M., Giumetti, G. W., Schroeder, A. N., \& Lattanner, M. R. (2014). Bullying in the digital age: a critical review and metaanalysis of cyberbullying research among youth. Psychological Bulletin, 140(4), 1073-1137. https://doi.org/10.1037/a0035618.

Manitoba Youth Health Survey Report//2012-2013. (2014). Retrieved from http://partners.healthincommon.ca/tools-and-resources/youthhealth-survey/. Accessed 2 April 2019.

Modecki, K. L., Minchin, J., Harbaugh, A. G., Guerra, N. G., \& Runions, K. C. (2014). Bullying prevalence across contexts: a meta-analysis measuring cyber and traditional bullying. Journal of Adolescent Health, 55, 602-611. https://doi.org/10.1016/j.jadohealth.2014.06. 007.

Morris, E. B., Zhang, B., \& Bondy, S. J. (2006). Bullying and smoking: examining the relationships in Ontario adolescents. Journal of 
School Health, 76(9), 465-470. https://doi.org/10.1111/j.17461561.2006.00143.x.

Nakamoto, J., \& Schwartz, D. (2010). Is peer victimization associated with academic achievement? A meta-analytic review. Social Development, 19(2), 221-242. https://doi.org/10.1111/j.14679507.2009.00539.x.

Napoletano, A., Elgar, F. J., Saul, G., Dirks, M., \& Craig, W. (2015). The view from the bottom: relative deprivation and bullying victimization in Canadian adolescents. Journal of Interpersonal Violence, 31(20), 3443-3463. https://doi.org/10.1177/0886260515585528.

Olweus, D. (2013). School bullying: development and some important challenges. Annual Review of Clinical Psychology, 9, 751-780. https://doi.org/10.1146/annurev-clinpsy-050212-185516.

Peleg-Oren, N., Cardenas, G. A., Comerford, M., \& Galea, S. (2012). An association between bullying behaviors and alcohol use among middle school students. Journal of Early Adolescence, 32(6), 761-775. https://doi.org/10.1177/0272431610387144.

Public Health Agency of Canada. (2016). The chief public health Officer's report on the state of public health in Canada 2015: alcohol consumption in Canada. Ottawa, ON. Retrieved from http:// healthycanadians.gc.ca/publications/department-ministere/statepublic-health-alcohol-2015-etat-sante-publique-alcool/alt/statephac-alcohol-2015-etat-aspc-alcool-eng.pdf. Accessed 22 March 2018.

Quinn, C. A., Fitzpatrick, S., Bussey, K., Hides, L., Chan, G. C. K., Beran, T., et al. (2016a). Bullying and smoking: examining the relationships in Ontario adolescents. Journal of Adolescent Health, 59(1), 6-13. https://doi.org/10.1177/0272431610387144.

Quinn, C. A., Fitzpatrick, S., Bussey, K., Hides, L., \& Chan, G. C. K. K. (2016b). Associations between the group processes of bullying and adolescent substance use. Addictive Behaviors, 62, 6-13. https://doi. org/10.1016/j.addbeh.2016.06.007.

Rosenthal, L., Earnshaw, V. A., Carroll-Scott, A., Henderson, K. E., Peters, S. M., McCaslin, C., \& Ickovics, J. R. (2015). Weight- and race-based bullying: health associations among urban adolescents. Journal of Health Psychology, 20(4), 401-412. https://doi.org/10. $1177 / 1359105313502567$.

Salmon, S., Turner, S., Taillieu, T., Fortier, J., \& Afifi, T. O. (2018). Bullying victimization experiences among middle and high school adolescents: traditional bullying, discriminatory harassment, and cybervictimization. Journal of Adolescence, 63(November 2017), 29-40. https://doi.org/10.1016/j.adolescence.2017.12.005.

Sánchez de Ribera, O., Trajtenberg, N., Shenderovich, Y., \& Murray, J. (2019). Correlates of youth violence in low- and middle-income countries: a meta-analysis. Aggression and Violent Behavior, 49(September), 101306. https://doi.org/10.1016/j.avb.2019.07.001.

Sigurdson, J., Wallander, J., \& Sund, A. (2014). Is involvement in school bullying associated with general health and psychosocial adjustment outcomes in adulthood? Child Abuse \& Neglect, 38, 1607-1617. https://doi.org/10.1016/j.chiabu.2014.06.001.

Stewart-Tufescu, A., Salmon, S., Taillieu, T., Fortier, J., \& Afifi, T. O. (2019). Victimization experiences and mental health outcomes among grades 7 to 12 students in Manitoba, Canada. International Journal of Bullying Prevention. https://doi.org/10.1007/s42380019-00056-0.

Takizawa, R., Maughan, B., \& Arseneault, L. (2014). Adult health outcomes of childhood bullying victimization: evidence from a fivedecade longitudinal British birth cohort. American Journal of Psychiatry, 171(7), 777-784. Retrieved from. https://doi.org/10. 1176/appi.ajp.2014.13101401.

Tucker, J. S., Ewing, B. A., Espelage, D. L., Green, H. D., de la Haye, K., \& Pollard, M. S. (2016). Longitudinal associations of homophobic name-calling victimization with psychological distress and alcohol use during adolescence. Journal of Adolescent Health, 59(1), 110 115. https://doi.org/10.1016/j.jadohealth.2016.03.018.

Turner, S., Taillieu, T., Fortier, J., Salmon, S., Cheung, K., \& Afifi, T. O. (2018). Bullying victimization and illicit drug use among students in grades 7 to 12 in Manitoba, Canada: a cross-sectional analysis. Canadian Journal of Public Health. https://doi.org/10.17269/ s41997-018-0030-0.

van Geel, M., Goemans, A., \& Vedder, P. H. (2015). The relation between peer victimization and sleeping problems: a meta-analysis. Sleep Medicine Reviews, 27, 89-95. https://doi.org/10.1016/j.smrv. 2015.05.004.

Vieno, A., Gini, G., \& Santinello, M. (2011). Different forms of bullying and their association to smoking and drinking behavior in Italian adolescents. Journal of School Health, 81(7), 393-399. https://doi. org/10.1111/j.1746-1561.2011.00607.x.

Volk, A. A., Dane, A. V., \& Marini, Z. A. (2014). What is bullying? A theoretical redefinition. Developmental Review, 34(4), 327-343. https://doi.org/10.1016/j.dr.2014.09.001.

Weiss, J. W., Mouttapa, M., Cen, S., Johnson, C. A., \& Unger, J. (2011). Longitudinal effects of hostility, depression, and bullying on adolescent smoking initiation. Journal of Adolescent Health, 48(6), 591-596. https://doi.org/10.1016/j.jadohealth.2010.09.012.

Wolke, D., \& Lereya, S. T. (2015). Long-term effects of bullying. Archives of Disease in Childhood, 100, 879-885. https://doi.org/ 10.1136/archdischild-2014-306667.

Wolke, D., Copeland, W. E., Angold, A., \& Costello, E. J. (2013). Impact of bullying in childhood on adult health, wealth, crime, and social outcomes. Psychological Science, 24(10), 1958-1970. https://doi. org/10.1177/0956797613481608.

World Health Organization. (2013). WHO | health effects of smoking among young people. 